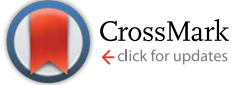

Cite this: RSC Adv., 2017, 7, 15978
Received 25th January 2017 Accepted 7th March 2017

DOI: 10.1039/c7ra01106g

rsc.li/rsc-advances

\section{Rheology of nitrile rubber with hybrid crosslinked network composed of covalent bonding and hydrogen bonding $\dagger$}

\begin{abstract}
Yonggang Shangguan, * Jie Yang and Qiang Zheng
A hybrid crosslinked network composed of covalent bonding and non-covalent bonding was constructed in nitrile rubber (NBR) by using the compound crosslinking agents dicumyl peroxide (DCP) and N,Nmethylenebis acrylamide (MBA). DCP not only acted as a chemical crosslinking agent for NBR but also initiated MBA association onto the rubber chains. In addition to the crosslinked network of covalent bonds, the acylamino groups of MBA could associate reversibly into junctions between rubber chains to form additional hydrogen bonds. It was found that the total crosslinking density of NBR increased with the increasing MBA amount. Both dynamic mechanical analysis and dynamic rheological measurement results indicated that samples with more hydrogen bonding were more sensitive to deformation and temperature. For the vulcanized NBR samples with MBA, their glass transition temperature was higher than those of vulcanized samples only with DCP and slightly increased with increasing MBA amount. The existence of hydrogen bonding led to a shorter linear viscoelastic region in vulcanized NBR samples with MBA. In addition, compared with the samples vulcanized with only DCP, the vulcanized samples containing MBA presented a higher storage modulus in the low frequency region but a lower one in the high frequency region. These results indicated that the hydrogen bonding induced by MBA decreased both the motion ability of the chain segments between the crosslinking points and the energy dissipation generated by internal friction of the polymer chain.
\end{abstract}

\section{Introduction}

As one of the most common polymeric materials applied in industry, rubber is widely used in automobiles, airplanes, etc. due to its excellent elasticity-rigidity balance. Since uncrosslinked rubbers usually present low strength and poor elasticity, it is difficult to meet the demand of many applications. As a result, vulcanized rubbers treated by sulphur, sulphur compounds or peroxides are more common and practical in our daily production and life. Generally speaking, the crosslinked network in vulcanized rubber is achieved by covalent bonds and consequently is an irreversible and permanent network unless degraded.

It is well-known that crosslinked structure in polymers can be classified into chemical crosslinked and physical crosslinked ones. Chemical crosslinking is a kind of junction tied by a covalent bond, ${ }^{1-4}$ electrovalent bond ${ }^{5-7}$ or coordinate bond ${ }^{8-11}$ while physical crosslinking is tied by a hydrogen bond, $\pi-\pi$ stacking, metal-ligand bond etc. ${ }^{\mathbf{1 2}-22}$ Under deformation,

MOE Key Laboratory of Macromolecular Synthesis and Functionalization, Department of Polymer Science and Engineering, Zhejiang University, Hangzhou 310027, China. E-mail: shangguan@zju.edu.cn

$\dagger$ Electronic supplementary information (ESI) available. See DOI: 10.1039/c7ra01106g physical crosslinked junctions usually dissociate prior to chemical crosslinked ones since their bond energies are generally lower than those of chemical crosslinking. After the deformation is removed, physical crosslinking could form again but the damage caused by the rupture of chemical crosslinking remains. ${ }^{4}$ Recently, many smart supermolecular materials with transient network by physical crosslinking have been reported, ${ }^{4,13,20,21,23-25}$ and they could present some special properties, such as self-healing, ${ }^{\mathbf{1 2 , 1 3 , 1 5 , 2 0 , 2 6}}$ stimuli responsiveness, ${ }^{22,27,28}$ toughness $^{\mathbf{2 9 , 3 0}}$ and adhesive. ${ }^{31-33}$

Transient network in polymers has been investigated since 1990s. ${ }^{34-38}$ In particular, the relaxation behavior of transient network was studied and several constitutive models were proposed on the basis of Maxwell model and Boltzmann superposition principle..$^{22,36,39,40}$ On shorter time scales than the junction breakage time $\left(\tau<\tau_{\mathrm{j}}\right)$, these physical crosslinks are associated and the polymer performs as an elastomer with a high modulus. Conversely, when $\tau>\tau_{\mathrm{j}}$, physical crosslinking points disassociate and the modulus decreases. ${ }^{34-38}$ There are roughly two ways to construct a transient network: one is attaching associative groups onto chains, such as urea, pyridine, hydroxyl, amino, aromatic group etc., which could associate each other by hydrogen bond, $\pi-\pi$ stacking or metalligand bond. ${ }^{20,22,25,32,40-42}$ By this means, some dual network gels containing covalent and hydrogen bonding interactions have 

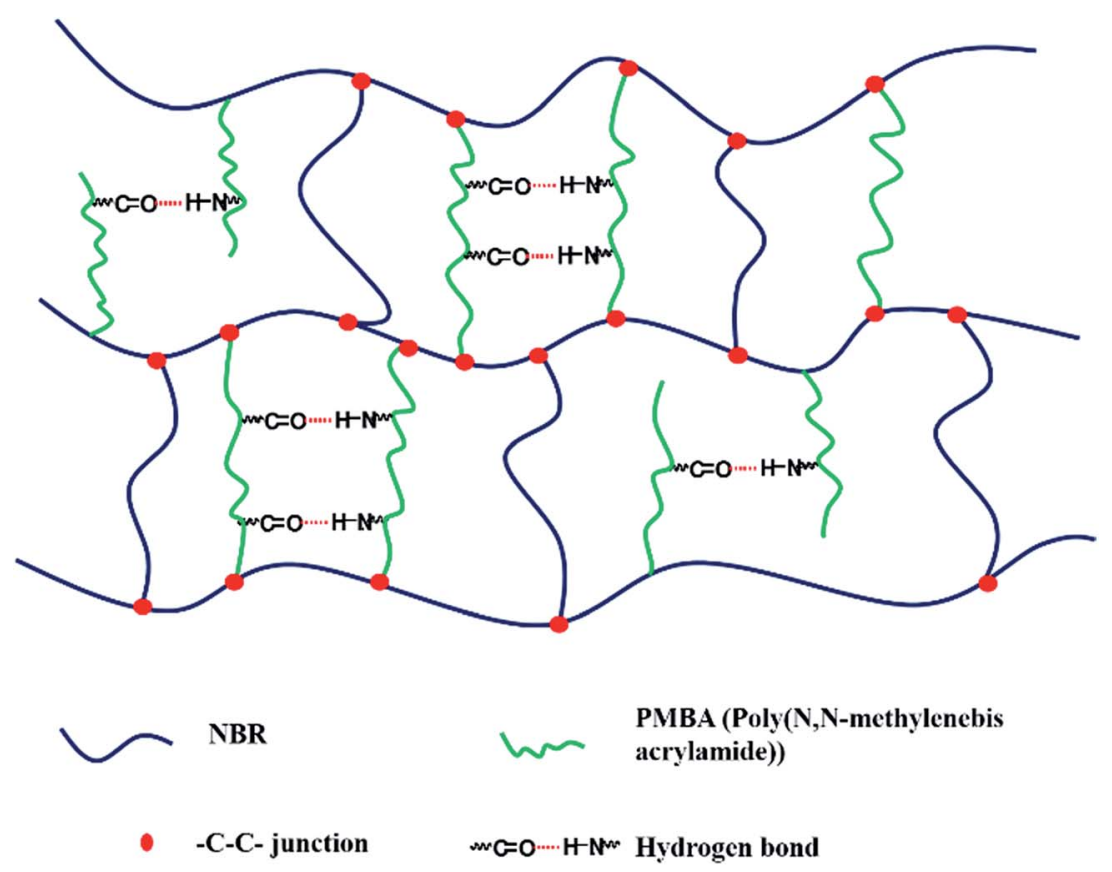

Scheme 1 Illustration of hybrid crosslinked network in NBR comprised of covalent bonding and hydrogen bonding.

been obtained..$^{23,43,44}$ The other way is by the exchange reaction of weak covalent bond between some groups. ${ }^{45,46}$ Non-covalent bond has attached great attention of researchers and some supermolecular materials have been prepared and explored. Sijbesma et $a .^{47}$ studied the dependences of modulus and critical strain on crosslinking density in a supermolecular gel. Tanaka et al. $^{\mathbf{4 0}}$ designed a transient polymer network crosslinked by two-component associative groups and studied the relaxation time of different junctions. Chung-Yuen et al. $^{\mathbf{4 8}}$ investigated the rate-dependent mechanical behavior and kinetics of breaking and reattachment of temporary crosslinks using a three-dimensional finite strain constitutive model. Weck et al. $^{22}$ studied the rheology of the polymer networks based on hydrogen bonding and metal coordination from a free-flowing liquid to a highly elastic gel and found that the dynamic moduli varied over 10 orders of magnitude. However, except constitutive model, most of researches just paid attention to the methods to construct a transient network and investigated its stimuli response or self-healing. Recently, Guo et al. ${ }^{\mathbf{1 7 - 1 9 , 4 9 , 5 0}}$ built networks via oxa-Michael reaction or metaloxygen coordination or hydrogen bond to endow the rubber materials with supermechanical performance or adaptive recovery or shape memory. In rheology, they investigated modulus response of transient network while barely explored the influence of temperature, deformation and frequency on viscoelasticity of transient network materials.

In this work, we try to establish a hybrid network in nitrile rubber (NBR) by using a compound vulcanizing agent of dicumyl peroxide (DCP) and $N, N$-methylenebis acrylamide (MBA). Polar nitrile group in NBR endows it excellent oil resistivity and consequently it has been widely applied in oil-resistive device. DCP is one of the most common peroxide crosslinking agents, which could decompose into radicals and crosslink polymers with active sites. MBA could be initiated by DCP and reacts onto NBR chain to construct a hybrid crosslinked network comprised of covalent bonding and hydrogen bonding as indicated by Scheme 1 , due to its two acylamino groups and two $-\mathrm{C}=\mathrm{C}-$. It is noticed that this network in NBR isn't a dual network system or interpenetrating networks, because it is mainly constructed by covalent bonds resulted from vulcanization and these hydrogen bonds only exist as additional crosslinking points. The crosslink density and glass transition behavior of vulcanized NBR samples with different DCP and MBA amounts were investigated. The influences of temperature, deformation and frequency on hybrid rubber network with two kinds of junctions are investigated by DMA and rheology measurement.

\section{Methods}

\subsection{Materials}

Nitrile rubber (NBR 3345C) with an acrylonitrile content of 33\% was purchased from Duo Kang, China. $N, N$-Methylenebis acrylamide (MBA 98\%) and dicumyl peroxide (DCP 99\%) were purchased from Aladdin Industrial Corporation.

\subsection{Sample preparation}

NBR were vulcanized by DCP or a complex vulcanizing agent of DCP and MBA, respectively. For the complex vulcanizing agent of DCP and MBA, DCP is not only crosslinking agent but it also act as initiator of MBA. The corresponding vulcanization mechanism is shown by Schemes 1 and 2 in ESI, $\dagger$ according to previous literatures. ${ }^{51-56}$

The formulation of NBR compounds for vulcanization in parts per hundred of rubber (phr) in weight is list in Table 1. These compounds were first mixed in internal mixer for $10 \mathrm{~min}$ 
Table 1 Formulation of NBR compounds for vulcanization

\begin{tabular}{llll}
\hline NBR $(\mathrm{phr})$ & DCP $(\mathrm{phr})$ & MBA $(\mathrm{phr})$ & Index \\
\hline 100 & 0.25 & 0 & DCP0.25/MBA0 \\
100 & 0.25 & 2 & DCP0.25/MBA0 \\
100 & 0.25 & 10 & DCP0.25/MBA10 \\
100 & 0.5 & 0 & DCP0.5/MBA0 \\
100 & 0.5 & 2 & DCP0.5/MBA2 \\
100 & 0.5 & 4 & DCP0.5/MBA4 \\
100 & 0.5 & 6 & DCP0.5/MBA6 \\
100 & 0.5 & 10 & DCP0.5/MBA10 \\
100 & 0.75 & 0 & DCP0.75/MBA0 \\
100 & 0.75 & 2 & DCP0.75/MBA2 \\
100 & 0.75 & 10 & DCP0.75/MBA10 \\
100 & 1 & 0 & DCP1/MBA0 \\
100 & 1 & 2 & DCP1/MBA2 \\
100 & 1 & 10 & DCP1/MBA10 \\
\hline
\end{tabular}

with a rotate speed of $60 \mathrm{rpm}$ at $70^{\circ} \mathrm{C}$. Subsequently, mixtures were laminated on an open two-roll mill with gap of $1 \mathrm{~mm}$ at $50{ }^{\circ} \mathrm{C}$ for $10 \mathrm{~min}$ for a sufficient mixing and then compressed at 140 centigrade and $10 \mathrm{MPa}$.

\subsection{Measurements}

2.3.1 Soxhlet extraction. To remove the unreacted residues of NBR, MBA and DCP, the vulcanized samples were extracted with acetone in Soxhlet extractor at $100{ }^{\circ} \mathrm{C}$ for $24 \mathrm{~h}$, and then the insoluble substance were dried in a vacuum oven at $50^{\circ}$ to constant weight. These extracted NBR were used to the next various measurements including elemental analysis, attenuated total reflection surface spectra, extension test and rheological measurements.

2.3.2 Elemental analysis. The elemental analysis gave out the ratio of $\mathrm{C}, \mathrm{H}, \mathrm{N}$ of samples with elemental analyzer (Vario Micro, Elementary Analysensysteme GmbH, China).

2.3.3 Swelling. After extracted with acetone in Soxhlet extractor at $100{ }^{\circ} \mathrm{C}$ for $24 \mathrm{~h}$, samples were swelled in acetone until a constant weight. Then the swellings were dried in a vacuum oven at $50^{\circ}$ to constant weight. The swelling degree, $Q$ is defined as below:

$$
Q=\frac{m_{\text {equi }}}{m_{\text {dry }}}
$$

in which, $m_{\text {equi }}$ is the weight of sample at swelling equilibrium, and $m_{\mathrm{dry}}$ is the weight of dried sample after swelling.

2.3.4 Extension test. Uniaxial tension was conducted on a universal testing machine (CMT 4204, Shenzhen SANS Test Machine Co. Ltd., China) at a tension rate of $500 \mathrm{~mm} \mathrm{~min}^{-1}$ at room temperature.

2.3.5 Attenuated total reflection surface spectra. ATR spectra of the thin film samples were obtained at ambient temperature with a Attenuated total reflection surface spectrometer (Nicolet 6700, Thermo Fisher scientific LLC, USA). The spectra of the samples were obtained after 32 scans between 4000 and $600 \mathrm{~cm}^{-1}$.

2.3.6 Rheological measurements. Dynamic mechanical properties were performed on a dynamic mechanical analysis (DMA Q800, TA, USA). For DMA investigations, a double cantilever mode was adopted with a dynamic strain of $100 \mu \mathrm{m}$. The samples were scanned from -60 to $120{ }^{\circ} \mathrm{C}$, under a frequency of $10 \mathrm{~Hz}$ and heating rate of $3{ }^{\circ} \mathrm{C} \mathrm{min}{ }^{-1}$.

Rheological measurements were performed on an advanced rheometric expanded system (ARES-G2, TA, USA), operated with $25 \mathrm{~mm}$ parallel plate setup. Strain $(\gamma)$ sweep in the range of $0.01-100 \%$ was performed. The frequency sweep was conducted from 100 to $0.01 \mathrm{rad} \mathrm{s}^{-1}$ at a constant strain of $0.1 \%$ which lies in linear region. For rheological tests, disk-like samples with 25 $\mathrm{mm}$ in diameter and $\mathbf{1 . 2} \mathrm{mm}$ in thickness were adopted.

\section{Results and discussion}

\subsection{Hybrid crosslinking networks}

Fig. 1 gives infrared absorption spectra of MBA, DCP, DCP0.5/ MBA10. The unreacted residues of NBR, MBA and DCP have been removed by Soxhlet extraction. Compared with MBA and DCP, characteristic peaks of aliphatic secondary amides appear in the spectrum of DCP0.5/MBA10. Peaks at $1666 \mathrm{~cm}^{-1}$ and $1540 \mathrm{~cm}^{-1}$ correspond to the absorption of $\nu_{\mathrm{C}=\mathrm{O}}$ (Amide I) and coupling effect of $\delta_{\mathrm{N}-\mathrm{H}}$ and $\nu_{\mathrm{C}-\mathrm{N}}$ (Amide II), respectively. In addition, the strong peak at $1626 \mathrm{~cm}^{-1}\left(\nu_{\mathrm{C}=\mathrm{C}}\right)$ in MBA becomes very weak and happens to disappear in DCP0.5/MBA10. It attests that MBA has reacted with NBR chain. As mentioned in Introduction section, MBA containing two acylamino groups and two $-\mathrm{C}=\mathrm{C}-$ is used to construct hydrogen bonding association in NBR matrix. Here, DCP is not only the initiator of MBA reaction but also directly crosslink NBR. Considering the self-polymerization tendency of MBA, MBA may exist in two different states: one is the chemical vulcanizing agent of NBR chains; another is the branched chain of NBR backbone, as shown in Scheme 1. However, both two states of MBA can form hydrogen bonding in vulcanized NBR and in turn leads to a hybrid crosslinked networks. Thus, to determine the crosslink density of vulcanized NBR is a primary issue.

Swelling equilibrium is a useful and simple method to determine the crosslink density of polymer network. The thermodynamic of swollen polymer networks is based on the FloryRehner model. ${ }^{5-59}$ Generally, the weight ratio of swollen network and dried network, $Q$, is used to characterize the crosslink density. The higher the value of $Q$, the lower crosslinking density is. Fig. 2 gives the dependence of $Q$ on MBA and

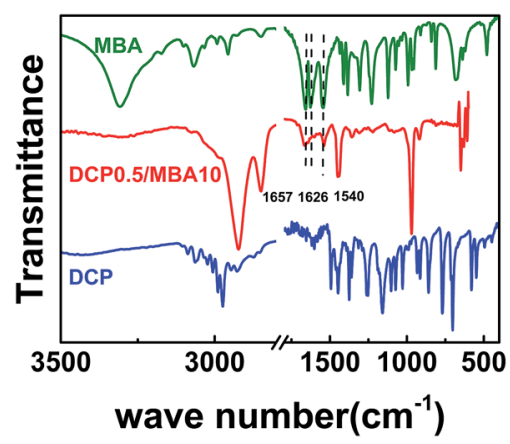

Fig. 1 Infrared absorption spectra of MBA, DCP, DCP0.5/MBA10. 


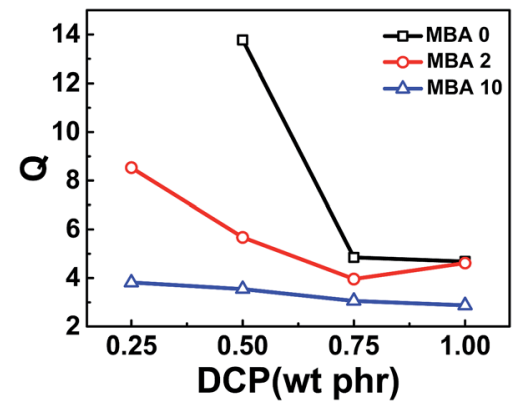

Fig. 2 Dependences of swelling degree of vulcanized NBR on MBA and DCP amounts in acetone at room temperature.

DCP amounts. It can be seen that $Q$ decreases with increasing MBA or DCP amount, indicating that the crosslink density increases with the increase of MBA or DCP. Furthermore, for vulcanized NBR samples with different MBA amounts, the difference of crosslink density gradually reduces with higher content of DCP. These results confirm that DCP not only directly crosslinks chains of NBR but also initiates the reaction of MBA and NBR. As mentioned above, considering that MBA may exist in two different states including the vulcanizing agent or branched chains of NBR backbone, the crosslink density results only indicate that both DCP and MBA contribute to crosslinking process, but couldn't provide more information about these two possible locations of MBA.

Fig. 3 shows DMA curves of various vulcanized NBR samples containing different DCP and MBA amounts, respectively. The loss factor, $\tan \delta$, is usually used to detect the relaxation behavior of different motion units of polymers. Generally speaking, the peak temperature is regarded as glass transition temperature $\left(T_{\mathrm{g}}\right)$ to characterize the motion of chain segment for amorphous polymer or chain segment between two crosslinking points for vulcanized systems. Furthermore, the value of $\tan \delta$ means internal friction degree between chains or the content of sol and damping chains. Thus, according to the plots of tan $\delta$ versus temperature or frequency, people can get in sight of the kinds of motion units and how it happens. As seen in Fig. 3, all NBR samples present only a single loss peak in the investigated temperature range. Obviously, this should be the glass transition of NBR. Fig. 3a gives the DMA of the samples vulcanized only by DCP. The curve of the unvulcanized sample also is presented. It can be found that for vulcanized samples, their loss peaks appear almost at the same temperature and are higher $3{ }^{\circ} \mathrm{C}$ than the unvulcanized sample, indicating that the DCP content has little influence on the motion ability of chain segment between crosslinking points. Considering that swelling test results mentioned above, i.e., crosslinking density of NBR samples increases with the increase of DCP amount, all NBR samples with different DCP amounts presenting the same $T_{\mathrm{g}}$ is interesting. These results may indicate that these vulcanized samples have similar chain length between crosslinking points. Fig. 3b gives DMA curves of the vulcanized NBR containing different MBA amounts. It is seen that for vulcanized NBR, their $T_{\mathrm{g}}$ increases with increasing MBA amount, but peak value decreases. The increased $T_{\mathrm{g}}$ indicates that the hydrogen bonding induced by MBA decreases the motion ability of chain segment between crosslinking points. Furthermore, the existence of hydrogen bonds decreases energy dissipation generated by internal friction of polymer chain, so the lower peak value appears in the vulcanized NBR with higher MBA content.

Fig. 4(a) gives stress-strain curves of vulcanized NBR samples with different MBA amounts. The vulcanized sample without MBA only presents an excellent toughness and the breaking elongation is more than $2000 \%$. As MBA amount increases, the breaking elongation of vulcanized samples with MBA decreases dramatically and the stress at definite elongation of $200 \%$ rises. Mooney-Rivlin model is often used to analyze the toughening and reinforcing properties of network in extension, ${ }^{\mathbf{6 0}}$ as followed below.

$$
\frac{\sigma}{\lambda-1 / \lambda^{2}}=2 C_{1}+\frac{2 C_{2}}{\lambda}
$$

here, $\sigma$ is engineering stress, $\lambda$ is stretch ratio, $C_{1}$ and $C_{2}$ is Mooney-Rivlin parameters.

According to Mooney-Rivlin equation, two parameters $2 C_{1}$ and $2 C_{2}$ can be estimated by plotting $\frac{\sigma}{\lambda-1 / \lambda^{2}}$ against $1 / \lambda$. Fig. 4(b) gives Mooney-Rivlin stress-strain curves of various NBR samples. At large strain $(1 / \lambda \rightarrow 0)$, the curves deviate the linear prediction and the more MBA, the earlier the deviation take places. $2 C_{1}$ is generally related to the chemical crosslinking of network and the value of $2 C_{1}$ is usually regarded as modulus, while $2 C_{2}$ is related to physical crosslinking. ${ }^{60-62}$ As the MBA content increases, $2 C_{1}$ increases, which means MBA increases
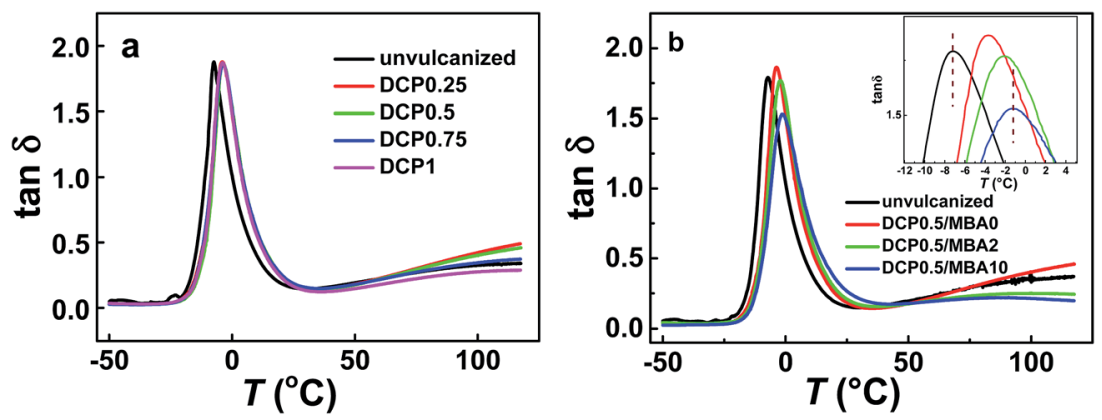

Fig. 3 (a) $\tan \delta$ of vulcanized NBR with different DCP amounts. (b) $\tan \delta$ of vulcanized NBR with different MBA content at 0.5 phr DCP. 
chemical crosslinking. But $2 C_{2}$ does not present some tendency. Since physical crosslinking consists of entanglement of long chains of sol, loops as well hydrogen bonding from MBA. Once MBA content increases, the content of sol and loops etc. decreases, resulting in the increase of hydrogen bonding and the decrease of entanglement. The two changes in physical crosslinking neutralize and there is no obvious trend in $2 C_{2}$. As a whole, the modulus of samples (in Table 2) increased with an increasing content of MBA except that the values of the samples containing MBA 6 phr and 10 phr are near, indicating that MBA increases crosslink density and reinforces the strength of the samples, which is in agreement with swelling experimental results.

To study the effect of MBA amount on vulcanized samples' properties, DCP amount was fixed at $0.5 \mathrm{phr}$. The amount and efficiency of MBA reacted with rubber chain are represented by reacting amount and reacting efficiency, respectively. Reacting amount $(R)$ is defined as the ratio of MBA amount in vulcanized sample after extraction in acetone and the weight of sample while reacting efficiency $\left(R_{\mathrm{e}}\right)$ is the ratio of the amount of MBA in sample after extraction in acetone and the feeding amount of MBA. Fig. 5 shows reacting amount and reacting efficiency of vulcanized samples calculated with the $D$-value of $\mathrm{N}$ element. The ratio of $\mathrm{C}, \mathrm{H}, \mathrm{N}$ of the samples was detected using an element analyzer. It can be found that the reacting ratio increases with the increase of MBA amount, while the reacting efficiency decreases. This may be related to the compatibility between MBA and NBR and the tendency of self-polymerization of MBA.

\subsection{Rheology of vulcanized NBR with hybrid crosslinked network}

To gain an insight into the structural understanding of sample network, a series of rheological tests were performed. Strain sweep of samples with $0.5 \mathrm{phr}$ DCP were tested at 40, 60, 80, and $100{ }^{\circ} \mathrm{C}$, respectively. As shown in Fig. 6a, all NBR samples at $80{ }^{\circ} \mathrm{C}$ present a linear viscoelastic region (LVER) in which storage modulus $\left(G^{\prime}\right)$ of samples is nearly a constant at low frequency range, indicating that the structure of samples remains in dynamic equilibrium. However, the frequency ranges of LVER for various samples are different, as indicated by
Table 2 Values of $2 C_{1}, 2 C_{2}$ obtained from linear fitting with MooneyRivlin equation

\begin{tabular}{lll}
\hline Sample & $2 C_{1}$ & $2 C_{2}$ \\
\hline DCP0.5/MBA0 & $0.0095 \pm 0.0033$ & $0.5600 \pm 0.0144$ \\
DCP0.5/MBA2 & $0.0385 \pm 0.0034$ & $0.6051 \pm 0.0273$ \\
DCP0.5/MBA4 & $0.1204 \pm 0.0095$ & $0.5242 \pm 0.0145$ \\
DCP0.5/MBA6 & $0.1014 \pm 0.0037$ & $0.5982 \pm 0.0267$ \\
DCP0.5/MBA10 & $0.2008 \pm 0.0106$ & $0.4855 \pm 0.0414$
\end{tabular}

the critical strain, $\gamma_{c}$ (estimated by the crossover strain of two linear fitted lines $)^{\mathbf{6 3 , 6 4}}$ in Fig. 6a, on the contrary, for vulcanized NBR samples only by DCP, their LVER are almost the same which are given in Fig. S2 in ESI. $\dagger$ The values of $\gamma_{c}$ for various samples with MBA at different temperatures are summarized in Fig. 6b. It is seen that $\gamma_{c}$ decreases with the increase of MBA amount. $\gamma_{c}$ of vulcanized samples increases as temperature rises. At $100{ }^{\circ} \mathrm{C}$, effect of MBA amount on $\gamma_{\mathrm{c}}$ becomes weak and the values of $\gamma_{c}$ for various samples are almost near. Furthermore, for the samples without MBA, $\gamma_{c}$ changes slightly. Generally speaking, the modulus of network is in proportion to the crosslinking density. It is easily understood that the junctions of NBR/DCP/MBA network include covalent bonds, physical bonds and entanglements of polymer chains. When a deformation or energy is applied, physical interactions and entanglements will be destroyed prior to covalent bonds. Since only chemical crosslinking exists in vulcanized NBR samples without MBA besides chain entanglement, the slip of $G^{\prime}$ should

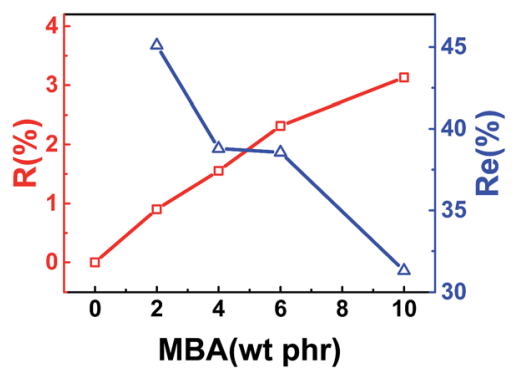

Fig. 5 Reacting amount $(R)$ and reacting efficiency $\left(R_{\mathrm{e}}\right)$ of vulcanized NBR versus MBA amount at $0.5 \mathrm{phr}$ DCP.
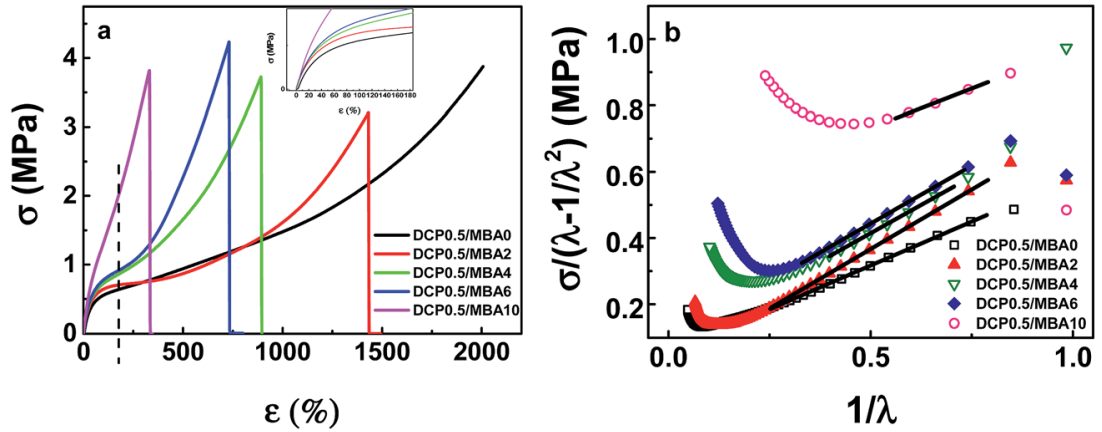

Fig. 4 (a) Stress-strain curves of vulcanized NBR samples with different MBA amounts. (b) Reduced stress-strain curves of NBR samples with different MBA amounts based on Mooney-Rivlin equation. 

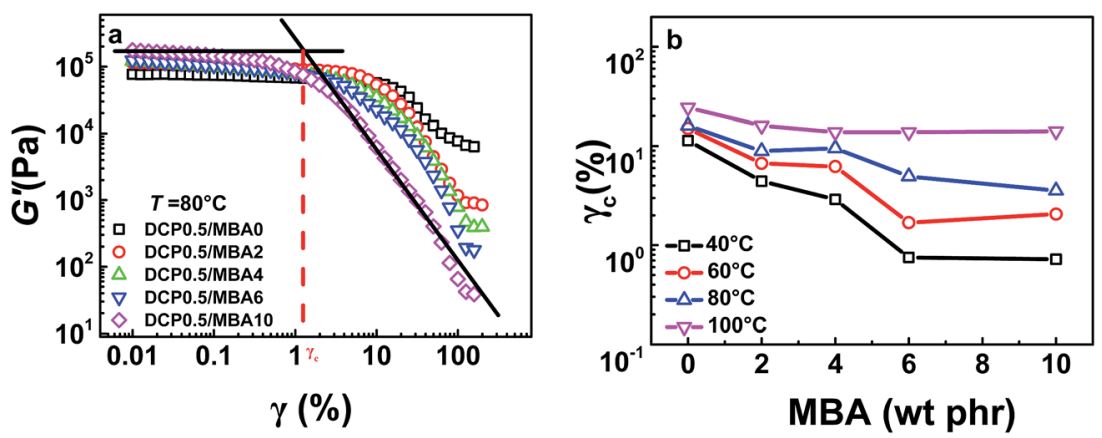

Fig. 6 (a) Strain sweep of samples with different MBA amounts at $80^{\circ} \mathrm{C}$

. (b) Critical strain $\gamma_{c}$ of samples at different temperatures.

be attributed to the disentanglement of matrix chain when frequency is higher than $10 \mathrm{~s}^{-1}$, which is insensitive to temperature. However, there are several hydrogen bonds in vulcanized samples with MBA and they are more sensitive to deformation and temperature. When they are subjected to a deformation or energy, these hydrogen bonds are dissociated first. As a result, the vulcanized NBR with higher MBA content are more sensitive to temperature and deformation and subsequently present a smaller $\gamma_{c}$, which implied that there were more physical crosslinks in these sample networks.

Fig. 7a gives the frequency sweep results of vulcanized samples with DCP at $80{ }^{\circ} \mathrm{C}$, which present typical crosslinked network behavior with storage modulus $\left(G^{\prime}\right)$ higher than loss modulus $\left(G^{\prime}>G^{\prime \prime}\right)$, as shown by the insert in Fig. 7a. The slope of $G^{\prime}$ for vulcanized NBR is much smaller than that of the unvulcanized sample and $G^{\prime}$ decreases slightly as angle frequency $\omega$ decreases, meaning the network of NBR is not perfect (the $G^{\prime}$ of a perfect network hardly changes with decreasing $\omega$ ). The vulcanized samples with MBA also present similar crosslinked network behavior. In addition, the vulcanized sample containing more DCP presents higher $G^{\prime}$ in low frequency region, which accords with the crosslink density results in Fig. 2, as described by affine network model, ${ }^{60}$ because the modulus in low frequency region mainly come from the contribution of crosslinked structure or entanglements of uncrosslinked chains. The affine network model is proposed as below:

$$
G=\nu \kappa T
$$

here, $v$ is crosslinking density, $\kappa$ is Boltzmann's constant and $T$ is temperature. Thus, $G^{\prime}$ increases linearly with the number density of network strands $\nu$. Note that although the sample with 0.25 DCP presents some feature of crosslinked structure in low frequency region, its $G^{\prime}$ curve in high frequency region is
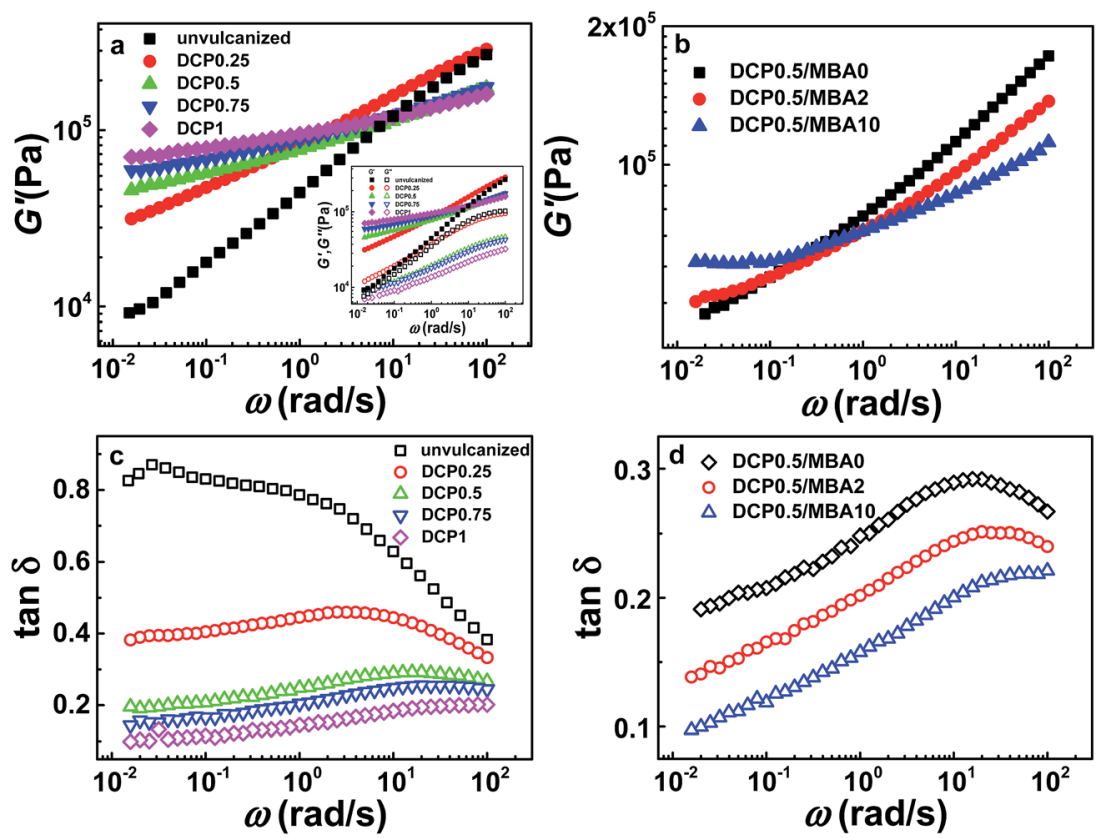

Fig. 7 (a) $G^{\prime}$ of samples vulcanized with different DCP amount versus angular frequency at $80^{\circ} \mathrm{C}$. (b) $G^{\prime}$ of vulcanized NBR samples with different MBA amount versus angular frequency at $80^{\circ} \mathrm{C}$. (c) tan $\delta$ of samples vulcanized with different DCP amount versus angular frequency at $80^{\circ} \mathrm{C}$. (d) $\tan \delta$ of vulcanized NBR samples with different MBA amount versus angular frequency at $80^{\circ} \mathrm{C}$. 
obviously different from other vulcanized samples and is closer to that of the unvulcanized sample. These results indicate that the crosslink density in the sample with 0.25 DCP is so lower that it has rheological behavior like the unvulcanized sample. For other vulcanized samples, their $G^{\prime}$ curve in high frequency region almost coincide, suggesting that the movement of chain segment between crosslinking points in them are similar, which is accordance with the results in Fig. 3a.

However, affine network model couldn't described the behavior of crosslinked systems containing both chemical and physical crosslinking, so a modification of Affine network model, Edwards model, is proposed as followed

$$
G=\left(\nu_{\mathrm{x}}+\nu_{\mathrm{e}}\right) \kappa T
$$

here, $\nu_{\mathrm{x}}$ is permanent crosslinking density, $\nu_{\mathrm{e}}$ is physical crosslinking density. Edwards model is more approximate to the experiment. ${ }^{63}$ Fig. $7 \mathrm{~b}$ gives frequency sweep results of vulcanized samples with different MBA amounts at $80^{\circ} \mathrm{C}$. It can be seen that the vulcanized sample with more MBA amount also has higher $G^{\prime}$ in low frequency region due to the increased crosslink density including chemical and physical junctions. However, $G^{\prime}$ of them in high frequency region differs from the case of vulcanized samples only containing DCP: the vulcanized sample with more MBA amount presents lower $G^{\prime}$. The frequency sweep results of these samples at other temperatures no more than $80{ }^{\circ} \mathrm{C}$ also are similar. Note that the chemical crosslinking density is rather low in vulcanized samples with lower MBA content and there are several amounts of sol and damping chains. In the high frequency region, although both uncrosslinked chains (including damping chains) and crosslinking contributes to $G^{\prime}$, contribution of the uncrosslinked chains is extremely significant and they couldn't relax in shorter time. As a result, $G^{\prime}$ of the vulcanized sample with less MBA is larger. Considering that the vulcanized samples only containing DCP present similar $G^{\prime}$ in high frequency region in Fig. 7a while vulcanized samples containing DCP and MBA present distinct $G^{\prime}$, it is suggested that the hybrid crosslinked network induced by MBA may decrease the distance between two crosslinking junctions, in other words, the chain segment between crosslinking points becomes shorter. Furthermore, it can be found from Fig. $7 d$ that the samples with MBA behave a smaller loss factor in the whole frequency range under investigation, which also indicates the samples with more MBA has more perfect network and less energy loss.

\section{Conclusions}

We constructed a hybrid crosslinking network in NBR with both covalent bonding and non-covalent bonding. The crosslink density of vulcanized samples remarkably increases with the increase of MBA amount. For the vulcanized NBR samples with MBA, their glass transition temperature is higher than those of vulcanized samples only with DCP and slightly increases with the increase of MBA amount while their $\tan \delta$ peak values decrease, indicating that the hydrogen bonding induced by MBA decreases both the motion ability of chain segment between crosslinking points and energy dissipation generated by internal friction of polymer chain. In addition, the existence of hydrogen bonding leads to a shorter linear viscoelastic region in vulcanized NBR samples with MBA. Although the vulcanized samples containing MBA presents higher storage modulus in low frequency region with the increase of MBA, the storage modulus in high frequency region decrease; on the contrary, $G^{\prime}$ curve of vulcanized samples only with DCP in high frequency region almost coincide. These results suggest that hybrid crosslinking network induced by MBA may decrease the distance between two crosslinking junctions and that the samples with more MBA has more perfect network and less energy loss.

\section{Acknowledgements}

This work was supported by National Nature Science Foundation of China (No. 51473145) and Zhejiang Provincial Natural Science Foundation of China (No. R16E030003). The authors are grateful for the discussion with Professor Chi Wu of The Chinese University of Hong Kong.

\section{References}

1 S. S. Choi and E. Kim, Polym. Test., 2015, 42, 62-68.

2 S. Mitra, A. Ghanbari-Siahkali, P. Kingshott, H. K. Rehmeier, H. Abildgaard and K. Almdal, Polym. Degrad. Stab., 2006, 91, 81-93.

3 D. M. Bieliński and A. Stępkowska, Arch. Civ. Mech. Eng., 2013, 13, 192-198.

4 F. Sordo, S. J. Mougnier, N. Loureiro, F. Tournilhac and V. Michaud, Macromolecules, 2015, 48, 4394-4402.

5 J. K. J. Stephen and C. W. Thomas, J. R. Soc., Interface, 2007, 4, 405-411.

$6 \mathrm{~W}$. H. Binder, Self-healing polymers: from principles to applications, Wiley-VCH, 2013.

7 S. D. Bergman and F. Wudl, J. Mater. Chem., 2008, 18, 41-62. 8 F. R. Kersey, D. M. Loveless and S. L. Craig, J. R. Soc., Interface, 2007, 4, 373-380.

9 J. R. Kumpfer, J. Jin and S. J. Rowan, J. Mater. Chem., 2010, 20, 145-151.

10 W. C. Yount, D. M. Loveless and S. L. Craig, J. Am. Chem. Soc., 2005, 127, 14488-14496.

11 M. Burnworth, D. Knapton, S. J. Rowan and C. Weder, J. Inorg. Organomet. Polym. Mater., 2007, 17, 91-103.

12 F. Herbst, D. Dohler, P. Michael and W. H. Binder, Macromol. Rapid Commun., 2013, 34, 203-220.

13 E. B. Stukalin, L. H. Cai, N. A. Kumar, L. Leibler and M. Rubinstein, Macromolecules, 2013, 46, 7525-7541.

14 B. A. Blight, C. A. Hunter, D. A. Leigh, H. McNab and P. I. Thomson, Nat. Chem., 2011, 3, 244-248.

15 Y. L. Chen, A. M. Kushner, G. A. Williams and Z. Guan, Nat. Chem., 2012, 4, 467-472.

16 J. Cortese, C. Soulie-Ziakovic, S. Tence-Girault and L. Leibler, J. Am. Chem. Soc., 2012, 134, 3671-3674.

17 Z. H. Tang, J. Huang, B. C. Guo, L. Q. Zhang and F. Liu, Macromolecules, 2016, 49, 1781-1789. 
18 J. Liu, S. Wang, Z. H. Tang, J. Huang, B. C. Guo and G. S. Huang, Macromolecules, 2016, 49, 8593-8604.

19 X. H. Zhang, Z. H. Tang, B. C. Guo and L. Q. Zhang, ACS Appl. Mater. Interfaces, 2016, 8, 32520-32527.

20 S. Burattini, B. W. Greenland, D. H. Merino, W. Weng, J. Seppala, H. M. Colquhoun, W. Hayes, M. E. Mackay, I. W. Hamley and S. J. Rowan, J. Am. Chem. Soc., 2010, 132, 12051-120589.

21 P. J. Woodward, M. D. Hermida, B. W. Greenland, I. W. Hamley, Z. Light, A. T. Slark and W. Hayes, Macromolecules, 2010, 43, 2512-2517.

22 K. P. Nair, V. Breedveld and M. Weck, Macromolecules, 2011, 44, 3346-3357.

23 D. Döhler, H. Peterlik and W. H. Binder, Polymer, 2015, 69, 264-273.

24 Y. P. Ni, F. Becquart, J. Chen and M. Taha, Macromolecules, 2013, 46, 1066-1074.

25 A. Gooch, C. Nedolisa, K. A. Houton, C. I. Lindsay, A. Saiani and A. J. Wilson, Macromolecules, 2012, 45, 4723-4729.

26 P. Cordier, F. Tournilhac, C. Soulie-Ziakovic and L. Leibler, Nature, 2008, 451, 977-980.

27 D. H. Merino, A. T. Slark, H. M. Colquhoun, W. Hayes and I. W. Hamley, Polym. Chem., 2010, 1, 1263.

28 V. Rajendran, B. W. Greenland, S. J. Rowan, H. M. Colquhoun, J. M. Elliott and W. Hayes, Macromolecules, 2012, 45, 5567-5574.

29 T. Aida, E. W. Meijer and S. I. Stupp, Science, 2012, 335, 813817.

30 S. Seiffert and J. Sprake, Chem. Soc. Rev., 2012, 41, 909-930.

31 C. R. Matos-Perez and J. J. Wilker, Macromolecules, 2012, 45, 6634-6639.

32 C. R. Matos-Perez, J. D. White and J. J. Wilker, J. Am. Chem. Soc., 2012, 134, 9498-9505.

33 G. Westwood, T. N. Horton and J. J. Wilker, Macromolecules, 2007, 40, 3960-3964.

34 L. Leibler, M. Rubinstein and R. H. Colby, Macromolecules, 1991, 24, 4701-4707.

35 F. Tanakat and S. F. Edwards, Macromolecules, 1992, 25, 1516-1523.

36 A. D. Drozdov, Acta Mechanica, 1999, 133, 13-37.

37 M. Muller, U. Seidel and R. Stadler, Polymer, 1995, 36(19), 3143-3150.

38 A. Dardin, H. W. Spiess, R. StadleI and E. T. Samulski, Polym. Gels Networks, 1997, 5, 37-54.
39 P. G. Khalatur, A. R. Khokhlov, J. N. Kovalenko and D. A. Mologin, J. Chem. Phys., 1999, 110, 6039.

40 T. Indei and F. Tanaka, J. Rheol., 2004, 48, 641.

41 C. A. Faler and M. M. Joullie, Org. Lett., 2007, 9, 1987-1990.

42 J. D. White and J. J. Wilker, Macromolecules, 2011, 44, 50855088.

43 R. Araya-Hermosilla, A. A. Broekhuis and F. Picchioni, Eur. Polym. J., 2014, 50, 127-134.

44 L. Rong, M. Koichi, C. Costantino, N. Tetsuharu and H. Chung-Yuen, J. Rheol., 2015, 59, 643-665.

45 H. Yang, K. Yu, X. Mu, X. Shi, Y. Wei, Y. Guo and H. J. Qi, Soft Matter, 2015, 11, 6305-6317.

46 G. Scheltjens, M. M. Diaz, J. Brancart, G. V. Assche and B. V. Mele, React. Funct. Polym., 2013, 73, 413-420.

47 M. M. E. Koenigs, A. Pal, H. Mortazavi, G. M. Pawar, C. Storm and R. P. Sijbesma, Macromolecules, 2014, 47, 2712-2717.

48 L. Rong, M. Koichi, C. Costantino, N. Tetsuharu and H. Chung-Yuen, Macromolecules, 2014, 47, 7243-7250.

49 T. F. Lin and B. C. Guo, Ind. Eng. Chem. Res., 2013, 52, 1812318130.

50 T. Lin, S. Ma, Y. Lu and B. C. Guo, ACS Appl. Mater. Interfaces, 2014, 6, 5695-5703.

51 J. L. Valentín, A. Rodríguez, A. Marcos-Fernández and L. González, J. Appl. Polym. Sci., 2005, 96, 1-5.

52 L. D. Loan, J. Appl. Polym. Sci., 1965, 7, 2259-2268.

53 L. Gonzalez, A. Rodriguez, A. Marcos and C. Chamorro, Rubber Chem. Technol., 1996, 69(2), 203-214.

54 L. Yang, B. Bai, C. Ding, H. Wang and Y. Suo, RSC Adv., 2016, 6, 9507-9517.

55 R. Suedee, V. Seechamnanturakit, B. Canyuk, C. Ovatlarnporn and G. P. Martin, J. Chromatogr. A, 2006, 1114, 239-249.

56 G. Y. Liu, X. L. Yang and Y. M. Wang, Polymer, 2007, 48, 4385-4392.

57 M. L. Huggins, J. Phys. Chem., 1942, 45, 151.

58 P. J. Flory, J. Chem. Phys., 1942, 10, 51.

59 P. J. Flory and J. Rehner, J. Chem. Phys., 1943, 11, 521.

60 M. Rubinstein and R. H. Colby, Polymer Physics, Oxford University Press, Oxford, 2011.

61 A. Roos and C. Creton, Macromolecules, 2005, 38, 7807-7818.

62 M. Rubinstein and S. Panyukov, Macromolecules, 2002, 25, 6670-6686.

63 C. Askling, L. Wågberg and M. Rigdahl, J. Mater. Sci., 1998, 33, 1517-1527.

64 H. Lutz, P. Sigrid and A. Nuri, Rheol. Acta, 2002, 41, 307-315. 